\title{
Estilos de liderazgo y satisfacción laboral de los enfermeros, Hospital Regional Virgen de Fátima, Perú
}

\section{Leadership styles and job satisfaction of nurses, Virgen de Fátima Regional Hospital, Peru}

\author{
Elito Mendoza Quijano' Sonia Tejada Muñoz ${ }^{2}$
}

\section{RESUMEN}

Investigación que tuvo como objetivo determinar la relación que existe entre estilos de liderazgo y satisfacción laboral de los enfermeros, Hospital Regional Virgen de Fátima, Perú, 2019. Se desarrolló mediante un enfoque cuantitativo de nivel relacional de tipología observacional, transversal y analítico, cuya población y muestra fue 72 enfermeros, que correspondió al 100\%, se utilizó el método hipotético- deductivo, el instrumento utilizado fue el cuestionario estilos de liderazgo con una confiabilidad de 0.75 por Alpha de Cronbach y el cuestionario de satisfacción laboral con una confiabilidad 0.79 por Alpha de Cronbach, se contrastó la hipótesis con Ji-cuadrado. Los resultados evidenciaron que los enfermeros encuestados declararon que en dicha institución los jefes y responsable del departamento de enfermería realizan un estilo de liderazgo transaccional (50\%) siendo este el más predominante, concerniente a la satisfacción laboral los enfermeros encuestados demostraron estar poco satisfecho (44\%) y comparando las dos variables antes mencionadas se encontró que el $25 \%$ de los enfermeros están satisfechos con el estilo de liderazgo transaccional. Se concluye que existe relación estadísticamente significativa entre estilos de liderazgo y satisfacción laboral por el valor de Ji-cuadrado: $\mathrm{X} 2=11.271, \mathrm{gl}=4, \mathrm{p}=$ $0.024<0.05$.

Palabras clave: Estilos, liderazgo, satisfacción laboral, enfermería

\section{ABSTRACT}

Research that aimed to determine the relationship between leadership styles and job satisfaction of nurses, Hospital Regional Virgen de Fátima, Peru, 2019. It was developed through a quantitative approach of relational level of observational, transversal and analytical typology, whose population The sample was 72 nurses, which corresponded to $100 \%$, the hypothetical-deductive method was used, the instrument used was the leadership styles questionnaire with a reliability of 0.75 by Cronbach's Alpha and the job satisfaction questionnaire with a reliability of 0.79 by Alpha of Cronbach, the hypothesis was contrasted with Chi-square. The results showed that the surveyed nurses declared that in this institution the heads and head of the nursing department carry out a transactional leadership style (50\%), this being the most predominant, concerning job satisfaction, the surveyed nurses showed little satisfaction ( $44 \%$ ) and comparing the two variables mentioned above, it was found that $25 \%$ of the nurses are satisfied with the transactional leadership style. It is concluded that there is a statistically significant relationship between leadership styles and job satisfaction by the value of Chi-square: $\mathrm{X} 2=11.271, \mathrm{gl}=$ $4, \mathrm{p}=0.024<0.05$.

Keywords: Styles, leadership, job satisfaction, nursing

\footnotetext{
${ }^{1}$ Maestro en Gerencia y Gestión en los Servicios de Salud. Docente de la Universidad Nacional Toribio Rodríguez de Mendoza de Amazonas. Email: elitoemq@gmail.com

${ }^{2}$ Doctora en Ciencias de Enfermería. Docente asociado a tiempo completo de la Universidad Nacional Toribio Rodríguez de Mendoza de Amazonas. Email: cieloceleste@hotmail.com
} 


\section{INTRODUCCIÓN}

El estilo de liderazgo es el modo o forma en el que lleva a cabo el proceso de persuasión o de ejemplo por medio del que un individuo (o equipo de liderazgo) induce a un grupo a alcanzar objetivos planteados por el líder o compartidos por el líder y sus seguidores, dentro de los estilos de liderazgo tenemos el transformacional, transaccional, Laissez Faire (Bass \& Avolio, 1990).

En todo el mundo el personal de enfermería conforma más del $60 \%$ de la fuerza de trabajo en salud y cubre el $80 \%$ de las necesidades de atención se evidencia que en España hay 50,8 enfermeros por cada 10.000 habitantes, otros países -como Australia, Finlandia, Alemania o Japón- tienen el doble (Organización Mundial de la Salud (OMS), 2015).

Chile tiene una ratio de 1,4 enfermeras por cada 10.000 habitantes, y aquellos con las ratios más elevadas como Canadá con 92,9 enfermeras por cada 10.000 habitantes (OMS, 2015).

Estados Unidos, Inglaterra, Escocia y Alemania reportaron un $60 \%$ de enfermeros que no había el suficiente personal de enfermería, la asociación de enfermeros de Massachusetts reporto que el $87 \%$ de sus colegas informaron que había demasiados pacientes propiciando así insatisfacción laboral (OMS, 2014).

En el Perú existe un batallón de 80.600 enfermeros, hay una densidad 12.8 enfermeros por 10,000 habitantes (Registro Nacional de Personal de salud (RNPS), 2015).

El profesional de enfermería tiene un pensamiento crítico y reflexivo, tiene la capacidad de incursionar en diversos ámbitos y ejercer un estilo de liderazgo con una visión amplia de certidumbre hacia el futuro, que favorece el clima organizacional y la satisfacción laboral (Quispe, 2015).

Es crucial que los enfermeros comprendan adecuadamente la financiación, los presupuestos, la asignación de recursos y la planificación estratégica de la atención de salud puesto que estas competencias facilitarán su participación en la formulación de políticas y la asignación de recursos para ser líderes desde la primera línea como fuerza para el cambio y como profesionales eficaces en los costos y eficaces en los cuidados (Consejo Internacional de Enfermería (CIE) 2015).

La satisfacción laboral es una sensación positiva sobre el trabajo propio, que surge de la evaluación de sus características, una persona con alta satisfacción en el trabajo tiene sentimientos positivos acerca de éste, en tanto que otra insatisfecha los tienen negativos (Robbins, 2009).

En un estudio realizado sobre el profesional de enfermería en 12 países europeos mostraron estar insatisfechos, manifestaron su intención de abandonar sus puestos de trabajo. La insatisfacción del profesional de enfermería estaba relacionada con los sueldos, las oportunidades de formación y la falta de progreso profesional (Aiken, 2015).

En Perú, los estudios dicen que los enfermeros asistenciales del Hospital Guillermo Almenara tienen insatisfacción laboral en $42.02 \%$ del personal de enfermería. En lo referente a la remuneración, ésta es desmotivante para $70 \%$. Para $3274 \%$ no satisface sus necesidades con la remuneración actual. El trabajo que realiza implica responsabilidad 95\%. Los ascensos por tiempo de servicio $72 \%$ afirma que no existe, los ascensos por habilidad $74 \%$ considera que no existe dichos ascensos (Flores, 2010).

En Lima sobre la satisfacción laboral que experimenta el enfermero durante el ejercicio de su profesión; un $60 \%$ de enfermeros experimentaron insatisfacción laboral, frente al $40 \%$ que sí mostraron satisfacción laboral, no encontrando porcentajes de enfermeros muy satisfechas. (Chávez \& Col, 2001).

En la región Amazonas especialmente en el distrito de Chachapoyas en un estudio realizado en el Hospital Regional Virgen de Fátima se encontraron resultados contundentes acerca de la satisfacción laboral del profesional de enfermería del $100 \%$ de los enfermeros encuestados correspondientes el $62.5 \%$ se encuentra regularmente satisfechos, $31.2 \%$ y se encuentran satisfechos (Bacalla, 2012).

La realidad sobre satisfacción laboral de los Profesionales de Enfermería muestran cifras inclinándose más hacia una insatisfacción condicionado por diferentes aspectos, el liderazgo como fuente principal de toda institución $\mathrm{u}$ organización está predispuesto a mediar teniendo en cuenta que el potencial humano es lo más importante con la que cuenta una organización es así que se planteó el siguiente problema de investigación ¿Qué relación existe entre estilos de liderazgo y satisfacción laboral de los enfermeros, Hospital Regional Virgen de Fátima, Perú - 2019?.

Se planteó las siguientes hipótesis Ha. Existe una relación estadísticamente significativa entre estilos de liderazgo y satisfacción laboral de los enfermeros, Hospital Regional Virgen de Fátima, Perú 2019. Ho. No existe una relación estadística entre estilos de liderazgo y Satisfacción Laboral de los enfermeros, Hospital Regional Virgen de Fátima, Perú- 2019. El Objetivo general fue: determinar la relación que 
existe entre estilos de liderazgo y satisfacción laboral de los enfermeros, Hospital Regional Virgen de Fátima, Perú - 2019. y los Objetivos Específicos: Identificar los estilos de Liderazgo y el nivel de satisfacción laboral de los enfermeros, Hospital Regional Virgen de Fátima, Perú 2019.

\section{MATERIAL Y METODOS}

\section{Enfoque, Nivel, Tipo y Diseño de investigación}

La presente investigación fue de enfoque cuantitativo, de nivel relacional de tipología observacional, prospectivo, transversal, analítico (Supo, 2016).

Diseño de la investigación:

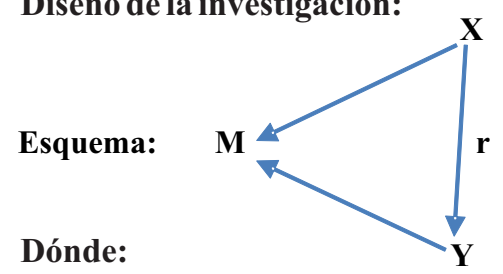

$\mathrm{M}=$ Enfermeros

$\mathrm{X}=$ Estilos de liderazgo

$\mathrm{Y}=$ Satisfacción laboral

$\mathrm{r}=$ Relación

Población y muestra de estudio: En función a los criterios de selección estuvo conformada por 72 enfermeros que laboran en el Hospital Regional Virgen de Fátima.

\section{Métodos de investigación}

En la presente investigación se utilizó el método hipotético-deductivo (Supo, 2016).

\section{Técnicas e Instrumentos de recolección de datos}

Se utilizó la técnica de la encuesta para las dos variables de estudio. (Supo, 2016).

Para medir la variable estilos de liderazgo se usó "EI Cuestionario Estilos de Liderazgo CELID-S" elaborado por Castro et al. (2004). Consta de 34 ítems que considera la teoría del Liderazgo Transformacional, Transaccional y Laissez faire de Bass (1990), Fiabilidad: 0.75. Alpha de Cronbach, Variable de naturaleza categórica medida en la escala ordinal teniendo como valor final un liderazgo transformacional como más favorable en una organización y menos favorable el transaccional y mucho menos favorable el laissez faire. Para medir la variable satisfacción laboral se utilizó la "El cuestionario de satisfacción laboral SL -SPC" elaborado por Palma, S. (1999), consta de 36 ítems y 7 dimensiones, condiciones físicas y/o confort, beneficios laborales y/o remunerativos, políticas administrativas, relaciones sociales, desarrollo personal, desempeño de tareas y relación con la autoridad. Fiabilidad: 0.79. Alpha de Cronbach, se midió con la escala ordinal teniendo como valor final, Satisfecho, Poco Satisfecho, Insatisfecho.

Análisis de datos

La información final fue procesada en el software SPSS versión 23, Microsoft Word y Excel, para contrastar la hipótesis se sometió a la prueba estadística no paramétrica de Ji-cuadrado con un nivel de significancia de $\alpha=0.05$ (95\% de nivel de confianza y un $5 \%$ de margen de error).

\section{RESULTADOS}

Tabla 1. Estilos de liderazgo y satisfacción laboral de los enfermeros.

\begin{tabular}{|c|c|c|c|c|c|c|c|c|}
\hline \multirow{3}{*}{ Satisfacción laboral } & \multicolumn{6}{|c|}{ Estilos de liderazgo } & \multirow{2}{*}{\multicolumn{2}{|c|}{ Total }} \\
\hline & \multicolumn{2}{|c|}{ Transformacional } & \multicolumn{2}{|c|}{ Transaccional } & \multicolumn{2}{|c|}{ Laissez Faire } & & \\
\hline & fi & $\%$ & fi & $\%$ & fi & $\%$ & fi & $\%$ \\
\hline Satisfecho & 9 & 12.5 & 18 & 25.0 & 0 & 0.0 & 27 & 37.5 \\
\hline Poco satisfecho & 16 & 22.2 & 12 & 16.7 & 5 & 6.9 & 33 & 45.8 \\
\hline Insatisfecho & 5 & 6.9 & 6 & 8.3 & 1 & 1.4 & 12 & 16.7 \\
\hline Total & 30 & 41.7 & 36 & 50.0 & 6 & 8.3 & 72 & 100.0 \\
\hline
\end{tabular}

$\mathrm{X}^{2}=11.271, \mathrm{Gl}=4, \mathrm{P}=0.024<0.05$ 
Tabla 2. Valores de estilos de liderazgo de los enfermeros

\begin{tabular}{lcc}
\hline Estilos de liderazgo & fi & $\%$ \\
\hline Transformacional & 30 & 41.7 \\
Transaccional & 36 & 50 \\
Laissez Faire & 6 & 8.3 \\
\hline Total & 72 & 100 \\
\hline
\end{tabular}

Tabla 3. Valores de la satisfacción laboral de los enfermeros.

\begin{tabular}{lcc}
\hline Satisfacción laboral & fi & $\%$ \\
\hline Satisfecho & 28 & 38.9 \\
Poco satisfecho & 32 & 44 \\
insatsifecho & 12 & 16.7 \\
\hline Total & 72 & 100 \\
\hline
\end{tabular}

\section{DISCUSIÓN}

En el presente estudio se puede identificar que el 50\% de los enfermeros que trabajan en el Hospital Regional Virgen de Fátima manifiestan que en dicha institución los jefes y responsables del departamento de la enfermería realizan un estilo de liderazgo transaccional, siendo este el de mayor porcentaje, seguido del estilo transformacional con el $41.7 \%$ y por ultimo estilo laissez faire con $8.3 \%$ respectivamente. (Tabla 2 ).

Esta investigación converge con los resultados Quenta (2016). Perú. Investigación que fue realizada con Enfermeros del Hospital Regional Honorio Delgado de Arequipa en donde encontraron como resultados que la mayoría de enfermeros identifican un liderazgo transaccional con un $74.5 \%$, seguido del liderazgo transformacional con $19.6 \%$ y en menor porcentaje identifican un liderazgo laissez faire con un $5.8 \%$.

Tal como se describe, el estilo de liderazgo transaccional es lo que predomina por parte de los enfermeros que trabajan en el Hospital Regional Virgen de Fátima lo cual se refiere a que los jefes y líderes optan por hacer una transacción con el personal trabajador para realizar las metas y objetivos planeados tal como se evidencia en la siguiente afirmación:

En el liderazgo transaccional el líder y seguidor aceptan roles y responsabilidades interconectados para alcanzar las metas designadas y acuerdan qué es lo que el subordinado debe hacer para obtener la recompensa (material o no material), o evitar la sanción estipulada en el contrato, los seguidores esperan que el líder los habilite para lograr un resultado favorable y sienten que el intercambio es transparente y justo si las recompensas son distribuidas equitativamente (Bass \& Avolio 1990).
Por otra parte, diverge con los resultados de Calderón (2016). Colombia. En su trabajo de investigación cuyo objetivo fue: identificar los estilos de liderazgo de los enfermeros supervisores y coordinadores de una Institución Prestadora de Servicios de Salud (IPS) del tercer nivel de la ciudad de Bogotá, los resultados evidenciaron: que el liderazgo que predomina en los enfermeros coordinadores y supervisores desde su percepción de los colaboradores es el liderazgo transformacional con un $39 \%$, en segundo lugar, el liderazgo transaccional con $38 \%$ y en tercer lugar, el liderazgo correctivo evitador con $23 \%$.

El líder transformacional provoca cambios en sus seguidores a partir de concientizarlos acerca de la importancia y el valor que revisten los resultados obtenidos tras realizar las tareas asignadas, no centra su atención solamente en la maximización del desempeño del individuo, sino que pone foco en la responsabilidad del integrante del grupo por su propio desarrollo personal, que como consecuencia trae un aumento en el desempeño del cumplimiento de los objetivos de la organización (Bass \& Avolio 1990).

Con respecto a la variable satisfacción laboral de los enfermeros que laboran en el Hospital Regional Virgen de Fátima, el $44.4 \%$ se encuentran poco satisfecho siendo este el mayor porcentaje, mientras que el $38.9 \%$ se encuentran satisfechos y el $16.7 \%$ se encuentran insatisfechos siendo este de menor porcentaje. (Tabla 3).

Esta investigación converge con los resultados de Bacalla (2012). Perú. En su trabajo cuyo objetivo fue: determinar la satisfacción laboral de los profesionales de enfermería de los Hospitales Virgen de Fátima -Chachapoyas y II-2 Tarapoto. Los resultados evidenciaron que del $100 \%$, de los profesionales de enfermería de los Hospitales Virgen de Fátima -Chachapoyas y II-2 Tarapoto el 62.5\% y $61.6 \%$ se encuentra regularmente satisfechos, $31.2 \%$ y $26.9 \%$ se encuentran satisfechos, el $2.1 \%$ y $7.7 \%$ manifiestan estar muy satisfechos y $4.2 \%$ y $3.8 \%$ manifiestan estar insatisfechos respectivamente.

También otras investigaciones evidencian los mismos resultados tales como la de Monzón (2007). Perú. Investigación que fue realizada con enfermeros que trabajan en el Hospital Regional Docente de Trujillo, respecto a la satisfacción laboral se evidencio que el $84.3 \%$ se encontraban poco satisfecho siendo esta el mayor porcentaje, mientras que el $11.4 \%$ manifestaron estar satisfechos y por ultimo solo el $4.3 \%$ estuvieron insatisfechos. Así mismo también los resultados de la investigación de Mamani (2013). Realizada con enfermeros que 
trabajan en la Micro red José Antonio Encinas de Puno, referente a la variable satisfacción laboral se evidencio que el $41.9 \%$ presentan estar poco satisfechos, mientras que el $32.3 \%$ están satisfechos y $25.8 \%$ están insatisfechos.

Estar satisfecho en el lugar de trabajo es importante en las diferentes áreas ya sea en el contexto físicas y/o confort, beneficios laborales y/o remunerativos, políticas administrativas, relaciones Sociales, desarrollo personal, desempeño de tareas, relación con la autoridad, pero también el estilo de liderazgo por parte de los jefes transita como fuente principal para el desempeño de los trabajadores, el profesional de enfermería debería de optar por un liderazgo transformacional que ponga en práctica y concientice a todos los enfermeros de una organización, este estilo de liderazgo basado en valores principios, en motivación ispiracional carisma o influencia idealizada, estimulación intelectual y consideración individualizada tal como se muestra en la siguiente definición:

En la actualidad se ha demostrado que el liderazgo transformacional tiene grandes beneficios si se aplica de manera constante, desarrollando al máximo las capacidades de los miembros que conforman el equipo de salud, logrando así el empoderamiento de la profesión de Enfermería en los diversos ámbitos establecidos (Quispe, 2015).

El presente estudio demostró que si existe relación entre estilos de liderazgo y la satisfacción laboral de los enfermeros que laboran en el Hospital Regional Virgen de Fátima, para contrastar la hipótesis se utilizó la prueba estadística no paramétrica de la Jicuadrado donde se obtuvo X2 $=11.271, \mathrm{Gl}=4, \mathrm{p}=$ $0.024<0.05$; lo cual indica la existencia de una relación estadísticamente significativa entre ambas variables. (Tabla 1).

Los datos evidenciaron que el 25\% presentan estar satisfechos con un estilo de liderazgo transaccional, siendo este el de mayor porcentaje y el $1.4 \%$ se encuentran insatisfecho con el liderazgo laissez faire, y no se encontró satisfechos con el estilo de liderazgo laissez faire, siendo estos del de menor porcentaje. Existe otras investigaciones que demuestran la relación que existe entre estas dos variables por lo que se menciona a continuación:

Gonzales (2013). Chile. En su trabajo de investigación cuyo objetivo fue: determinar si la satisfacción laboral percibida por los profesionales de enfermería de los Centros de Responsabilidad (CR) o Servicios de 5 Hospitales del sector "Gran Santiago", de la Región Metropolitana, está relacionada a los estilos de liderazgo del Modelo de
Hersey y Blanchard. Se concluyó que: hay una relación estadísticamente significativa entre la satisfacción laboral con los estilos de liderazgo, se contrasto la hipótesis mediante ji-cuadrado X2= $26,293 \mathrm{Gl}=3(\mathrm{p}<0.05) \mathrm{p}=0,001$ motivo por el cual es importante profundizar en estos temas para obtener conocimientos que permitan formar profesionales líderes, capaces de mantener satisfecho a su equipo de trabajo.

Quenta (2016). Perú. En su trabajo de investigación cuyo objetivo fue: establecer la relación entre los estilos de liderazgo y la satisfacción laboral en enfermeras del Hospital Regional Honorio Delgado, Arequipa-2016. Concluyeron que con la aplicación de prueba estadística Ji-cuadrado, con un nivel de confianza al $99 \%$ y un nivel de error del $1 \%$, se encontró un alto nivel de significancia X2 $=25.63>$ $\mathrm{X} 2(99 \%)=20.09(\mathrm{P}<0.01) \mathrm{p}=0.0012$ entre ambas variables por lo que se acepta la hipótesis que señala que existe relación entre los estilos de liderazgo y satisfacción laboral de los enfermeros del Hospital Regional Honorio Delgado.

Carrera (2014). Perú. En su estudio cuyo objetivo fue: determinar qué relación existe entre la capacidad de liderazgo y el nivel de satisfacción laboral en los enfermeros de la Red Pacasmayo 2014. Lo cual concluyeron que la capacidad de liderazgo tiene relación significativa con el nivel de satisfacción laboral de los profesionales de enfermería, siendo comprobado por la prueba de Ji-cuadrado. X2 = $8.499(\mathrm{p}<0.05) \mathrm{p}=0.014$ significativo.

Los estilos de liderazgo y satisfacción laboral repercuten y se relacionan es por ello que los profesionales de enfermería que desempeñan cargos como direcciones: jefaturas de departamento, jefes y/o responsables de cada servicio deben de formarse y practicar un estilo de liderazgo que sea acorde con la satisfacción de todo el personal enfermero de la institución.

\section{CONCLUSIONES}

Existe relación significativa entre estilos de liderazgo y satisfacción laboral de los enfermeros que laboran en el Hospital Regional Virgen de Fátima- Perú, se contrastó la hipótesis con la prueba no paramétrica de Ji-cuadrado cuyo valor fue de: $\mathrm{X} 2=11.271, \mathrm{Gl}=4$, $\mathrm{p}$ $=0.024<0.05$, además se encontró que en dicho Hospital es predominante un estilo de liderazgo transaccional $(50 \%)$ por parte de los jefes y responsable del departamento de enfermería, correspondiente a la satisfacción laboral los enfermeros manifestaron estar poco satisfecho el $(44 \%)$ 


\section{REFERENCIAS BIBLIOGRAFICAS}

Aiken, R. (2015). Satisfacción Laboral e intención de abandonar la organización en personal de enfermería. Consultado el 18 de mayo del $\begin{array}{lllll}2 & 0 & 1 & 9\end{array}$. http://biblioteca2.ucab.edu.ve/anexos/bibliot eca/marc/texto/AAT1201.pdf

Bass, B., Avolio, B. (1990). Adaptación del cuestionario multifactorial de liderazgo. Consultado el 22 de Julio del 2019 lhttp://repositorio.uchile.cl/tesis/uchile/2004 /vega_c/sources/vega_c.pdf

Bacalla, K. (2012). Satisfacción laboral de los profesionales de enfermería. Hospitales Virgen de Fátima - Chachapoyas y II-2 Tarapoto - 2012 (Tesis de Licenciatura. Universidad Nacional Toribio Rodríguez de Mendoza de Amazonas). Repositorio institucional. Consultado el 23 de junio del $\begin{array}{llll}2 & 0 & 1 & 9\end{array}$ http://repositorio.untrm.edu.pe/handle/UNT $\mathrm{RM} / 20$

Carrera, F. (2014). Capacidad de liderazgo y nivel de satisfacción laboral de los profesionales de enfermería de la red de salud Pacasmayo 2014 (Tesis de licenciatura. Universidad Nacional de Trujillo). Repositorio institucional. Consultado el 11 de julio del $\begin{array}{llll}2 & 0 & 1 & 9\end{array}$. http://dspace.unitru.edu.pe/handle/UNITRU $/ 854$

Calderón, S. (2016). Estilos de liderazgo de las enfermeras coordinadoras y supervisoras de una IPS de tercer nivel de Bogotá (Tesis de Maestría. Universidad Nacional de Colombia). Repositorio institucional. Consultado el 19 de agosto del 2019. http://bdigital.unal.edu.co/56025/1/1022363 661.2016.pdf

Canta, L. (2016). Nivel de motivación y satisfacción laboral de los profesionales de Enfermería en el Hospital Regional Virgen de Fátima -Chachapoyas, 2016 (Tesis de Maestría. Universidad Nacional Toribio Rodríguez de Mendoza de Amazonas). Repositorio institucional. Consultado el 25 de setiembre d e $1 \quad 2019$. http://repositorio.untrm.edu.pe/bitstream/ha ndle/UNTRM/1319/Luzlita\%20Canta $\% 20 \%$ 20Zumaeta.pdf? sequence $=1 \&$ isAllowed $=y$

Consejo Internacional de Enfermería. (2015). Las enfermeras, una fuerza para el cambio.
Consultado el 20 de agosto del 2019. http://www.codem.es/reportajes/enfermeras-una-fuerza-para-cambio

Chávez, M., Col, C. (2001). Motivación, Satisfacción, Enfermera Asistencial. Consultado el 08 de setiembre del 2019 http://gesdoc.isciii.es/gesdoccontroller?actio $\mathrm{n}=$ download\&id=21/05/2014-e7053fe $25 \mathrm{f}$

Flores, A. (2010). Indicadores de satisfacción laboral en lima Perú. Consultad o el 15 de set i e mbre del 2019. http://gesdoc.isciii.es/gesdoccontroller?actio n=download\&id=21/05/2014-e7053fe $25 f$

Gonzales, L. (2013). Relación de la satisfacción laboral con estilos de liderazgo en enfermeros de hospitales públicos, (Tesis de maestría. Universidad de Chile). Repositorio institucional. Consultado el 14 de mayo del $\begin{array}{llll}2 & 0 & 1 & 9\end{array}$ http://www.redalyc.org/pdf/3704/370441812 002.pdf

Monzón, D. (2007). Capacidad de liderazgo y nivel de satisfacción laboral Trujillo (Tesis de licenciamiento. Universidad Nacional de Trujillo). Repositorio institucional. Consultado el 28 de agosto del 2019. http://www.nutricionhospitalaria.com/pdf/46 88.pdf

Organización Mundial de la Salud. (2015). Las enfermeras, una fuerza para el cambio: eficaces en cuidados, eficientes en costos. Consultado el 01 de enero del 2020. http://files.sld.cu/socuenf/files/2015/04/carpe ta-del-die_2015_sp1.pdf

Organización Mundial de la Salud. (2014). Reposte a nivel mundial sobre el entorno laboral del profesional de enfermeria: eficaces en cuidados, eficientes en costos. Consultado el $13 \mathrm{~d}$ e $\quad$ o c t u b r e d e 122019 . http://files.sld.cu/socuenf/files/2014/04/carpe ta-del-die_2014_sp1.pdf

Quenta, R. (2016). Estilos de liderazgo y satisfacción laboral en enfermeras del hospital regional Honorio delgado Arequipa - 2016, (Tesis de Licenciatura. Universidad Nacional de San Agustín de Arequipa). Repositorio institucional. Consultado el 02 de marzo del $\begin{array}{lccc}2 & 0 & 1 & 9 \\ \text { http://repositorio.unsa.edu.pe/bitstream/hand } \\ \text { le/UNSA/1805/ENquvara.pdf?sequence }=1\end{array}$.

Quispe, J. (2015). Estilos de liderazgo y satisfacción laboral de enfermeros del hospital regional 
Honorio Delgado, Tesis de Licenciatura. Universidad Nacional Mayor de San Marcos. Perú. Consultado el 20 de enero del 2019. http://cybertesis.unmsm.edu.pe/bitstream/cy bertesis/4107/1/Quispe_\%C3\%

Registro Nacional del Penal de Salud. (2015). Número de profesionales de salud creció en más de 27,000 en último quinquenio. Consultado el 25 de febrero del 2019.http://www.upch.edu.pe/faest/images/s tories/egresados/pdf/1612-1.pdf

Robbins, S. (2009). Estilos de Liderazgo. Consultado el 03 de marzo del 2019. http://catarina.udlap.mx/u_dl_a/tales/docum entos/lad/pena_c_fj/bibliografia.pdf

Supo, J. (2016). Seminarios de investigación científica. Consultado el 05 de enero del 2019. file:///C:/Users/PC/Downloads/107josc3a9-supo-cc3b3mo-empezar-unatesis.pdf 